\title{
CARACTERIZACIÓN MORFOMÉTRICA DE FORMAS GLACIARES EN CUÑA EN LAS SIERRAS DE XISTRAL, TELENO Y CABRERA
}

\author{
Jonathan F. HALL-RIAZA (jonathanfernando.hall@rai.usc.es) \\ Departamento de Geografía, Universidad de Santiago de Compostela \\ Marcos VALCÁRCEL (marcos.valcarcel@usc.es) \\ Departamento de Geografía, Universidad de Santiago de Compostela \\ Ramón BLANCO-CHAO (ramon.blanco@,usc.es) \\ Departamento de Geografía, Universidad de Santiago de Compostela
}

Recibido: $27 / 01 / 2016$

Aceptado: 21/04/2016

RESUMEN: En el presente trabajo se cita la presencia en distintos macizos del NW de la Península Ibérica de formas de erosión glaciar descritas como «formas glaciares en cuña». Este tipo de formas se describen en la bibliografía como vinculada a glaciares activos, y se consideran indicadoras de la presencia de glaciarismo de base fría. Estas condiciones indican en último término la presencia de permafrost continuo. Otros indicios, como la presencia en el área de estudio de campos de bloques refuerzan esta interpretación.

PALABRAS CLAVE: Breitböden, Formas glaciares en cuña, Teleno, Cabrera, Xistral, Pleistoceno.

MOPHOMETRIC CHARACTERIZATION OF «BREITBÖDEN》 GLACIERS LANDFORMS IN THE XISTRAL, TELENO AND CABRERA RANGES

ABSTRACT: This study focuses on the presence of glacial erosion features, known as «breitböden» glaciers landforms, in different mountain areas of the NW of the Iberian Peninsula. These types of erosion landforms are produced by glaciers, and are considered indicators of the presence of cold based glaciers. These conditions indicate, therefore, the need for continuous permafrost for their development. Other signs such as the presence of blockfields reinforce this interpretation.

KEY WORDS: Breitböden, Glacial landforms, Teleno, Cabrera, Xistral, Pleistocene.

\section{INTRODUCCIÓN}

HOFMANN (1992) y LEHMKUHL (1991) señalan que el término «breitböden», traducido aquí por «forma glaciar en cuña», fue introducido por HÖVERMANN (1974) para describir las formas de erosión glaciar origina- 
das por antiguos glaciares de escape en el ámbito del Acker-Bruchberg (montañas Harz) (FIGURA 1). Se trataría de «... superficies ensanchadas triangulares que convergen hacia abajo, hundiéndose marcadamente en su parte inferior, mientras que en su parte superior se fusionan de forma más o menos marcada con su entorno. Los límites laterales normalmente están definidos por pequeños arroyos que se encuentran algo incididos, quedando muy marcado el borde con la ladera vecina. Pero también pueden ser, dichos surcos, muy poco marcados, o estar totalmente ausentes» (HöVERMANN, 1974). Según LEHMKUHL (1991), y a diferencia de los circos glaciares, los breitböden no presentan sobreexcavación y sus medidas oscilan entre los 0,5 y los $2,5 \mathrm{~km}$ en su parte más ancha.

Figura 1. Breitböden de Harz descritos por Hövermann.

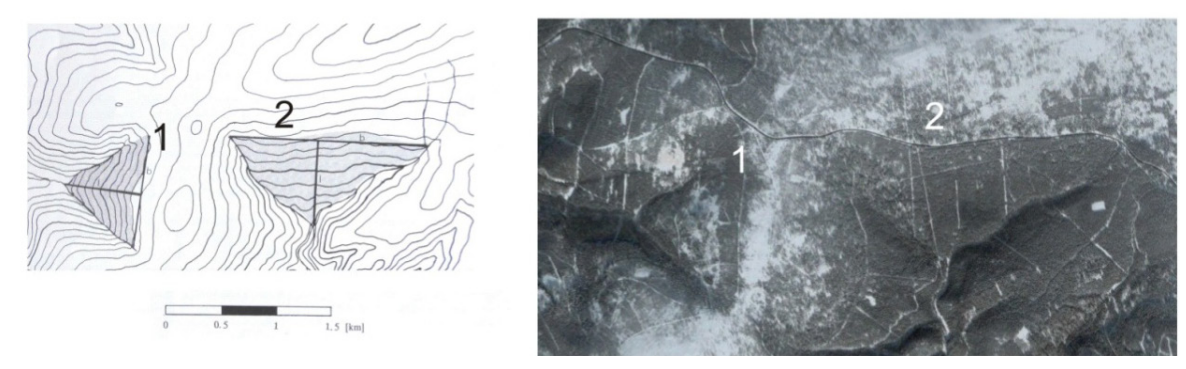

FUENTE.: LEHMKUHL, 1991.

Estas formas «breitböden» fueron definidas, pues, como formas de erosión glaciar, y se han descrito como formas fósiles en las montañas Tabai (RosT, 1993, 1994, 2000; Rost et al., 1991) y Helan (HoFMANN, 1992); y como formas activas en Spitzberg, norte de Canadá (HöVERMANN, 1974: 50), en las montañas Quilian y en el Kumlun (FIGURA 2) (LEHMKUHL, 1991), recibiendo los glaciares asimilables a estas formas la denominación de «breitbodengletscher», traducido aquí como "glaciares en cuña», y siendo para este último autor una variedad de glaciares de pared, según la tipología de Schneider (1963). El hecho de haber sido descritos como formas actuales permite definir las condiciones ambientales en las que son activos. En su momento fueron clasificados como glaciares «fríos» relacionados con condiciones climáticas frío-áridas (HÖVERMANN, 1974). Las observaciones sobre breitbodengletscher coinciden con esta apreciación, con temperaturas estimadas para la línea de equilibrio glaciar (ELA) que oscila, en los glaciares actuales, entre los $-8,5^{\circ} \mathrm{C}$ en el Kunlun y $\operatorname{los}-7,9^{\circ} \mathrm{C}$ en las montañas Qilian (LEHMKUHL, 1991). De esta manera, la presencia de formas de este tipo sería un posible indicador de glaciares de base fría, donde la temperatura del hielo en el lecho del glaciar queda por debajo del punto de fusión por presión (WILHELM, 
1975). Esto explicaría la profusión de agua en superficie que se canalizaría por los laterales de la lengua glaciar en forma de triángulo invertido.

Figura 2. Breitböden en Kunlun oriental (alrededores del pico Yuzhu Feng de 6178 m, Provincia de Qinghai, República Popular de China).

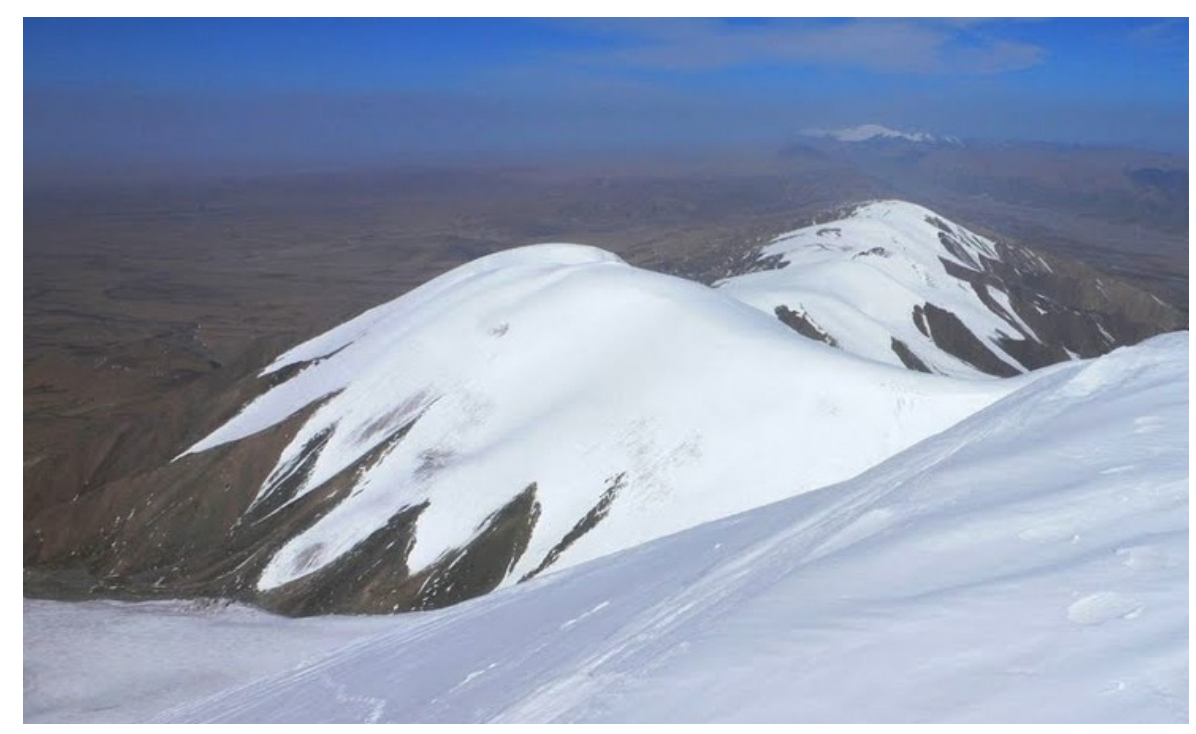

FUENTE.: Fotografía cedida por Pedro Ibarra.

Figura 3. Breitböden (BXI) de la Serra de Xistral.

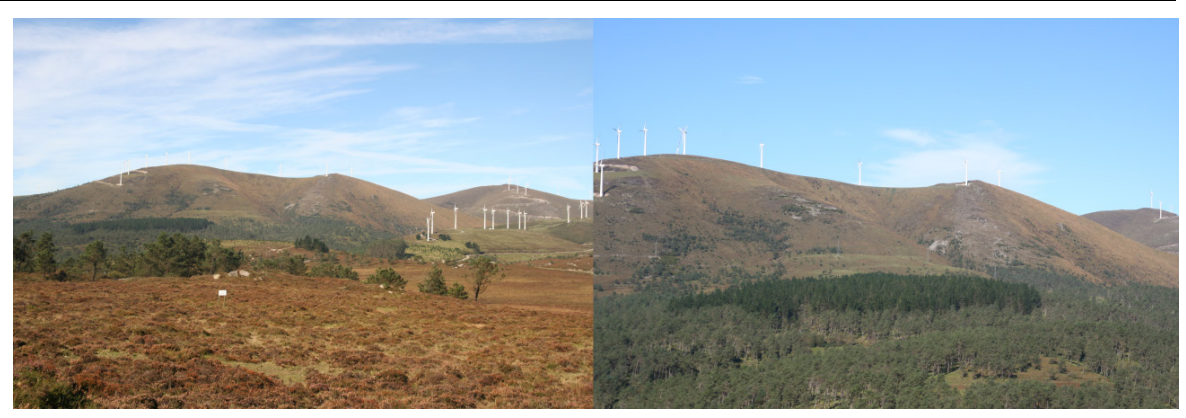

FUENTE.: Los autores.

Descritas tanto como formas activas y a partir de formas de erosión generadas en áreas afectadas por frío pleistoceno, estas formas han sido identificadas como ejemplos de este segundo caso en tres áreas de montaña del Noroeste peninsular, a saber: Serra do Xistal (FIgURAS 3 y 4), Sierra de Teleno y Sierra 
de la Cabrera. El objeto del presente trabajo es describirlas desde el punto de vista morfométrico, intentando establecer una tipificación de las mismas.

Figura 4. Breitböden de la Serra de Xistral. A: Fotografia aérea tomada de Google Earth. B: Imagen tomada desde orientación N. C: Imagen tomada desde orientación noroeste.

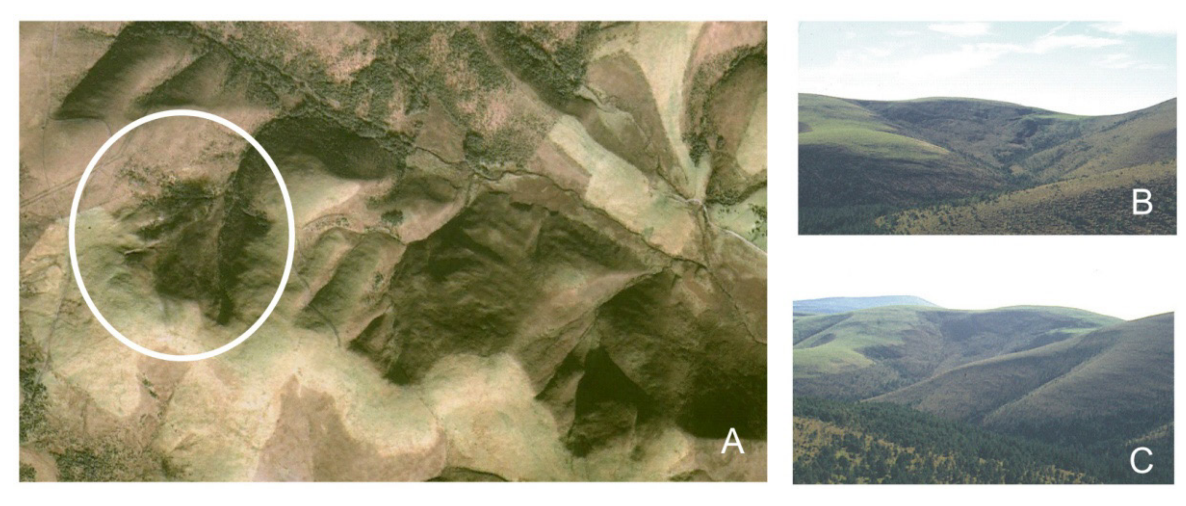

FUENTE.: Los autores.

\section{AREA DE ESTUDIO}

Las Sierras Septentrionales de Galicia se corresponden con un conjunto montañoso de modesta altitud, ya que en conjunto superan escasamente los $1.000 \mathrm{~m}$, siendo su núcleo la Serra de Xistral. En este macizo se distingue claramente un escalonamiento a partir de la sucesión de diferentes niveles aplanados, de los cuales el más elevado se sitúa sobre los $900 \mathrm{~m}$. Desde éste se elevan, a modo de picos aislados, las cimas más altas (Chan de Lamoso, 1.039 m; Xistral, 1.036; Seixo Blanco, 1.057 m; Cadramón, 1.060 m; Lombo Pequeno, $1.036 \mathrm{~m}$ ). Litológicamente destaca la presencia de las Cuarcitas de Xistral, de edad Cámbrico inferior, y que dominan totalmente las áreas cimeras, aunque también están presentes en este sector las filitas, micaesquistos y gneises anfibólicos, así como afloramientos graníticos. Todos ellos han sido afectados durante diferentes momentos de su evolución geológica por una intensa fracturación (FIGURAS 5 y 6 ).

Por su parte, la Sierra del Teleno forma parte de los Montes de León actuando, junto con los Montes Aquilianos, que son su prolongación natural, como reborde sur de la Cuenca del Bierzo. Sus cumbres superan los $2.000 \mathrm{~m}$, siendo el propio pico Teleno su cúspide $(2.183 \mathrm{~m})$. Desde él drenan las aguas del Duerna por su cara norte y las del Eria por su flanco sur. Los materiales geológicos que la arman son las Cuarcitas Armoricana de sus cumbres, que en las laderas pasan a intercalaciones de pizarras. Del mismo modo, este tipo de 
materiales afloran también en la alineación de la Sierra de la Cabrera, una divisoria de aguas que drena el Tera hacia el sur, mientras que su vertiente norte circulan las aguas de diferentes arroyos y ríos de montaña que van a unirse al Eria (FIGURAS 5,7 y 8).

Figura 5. Zonas de estudio 1. Xistral, 2. Teleno, 3. Cabrera.

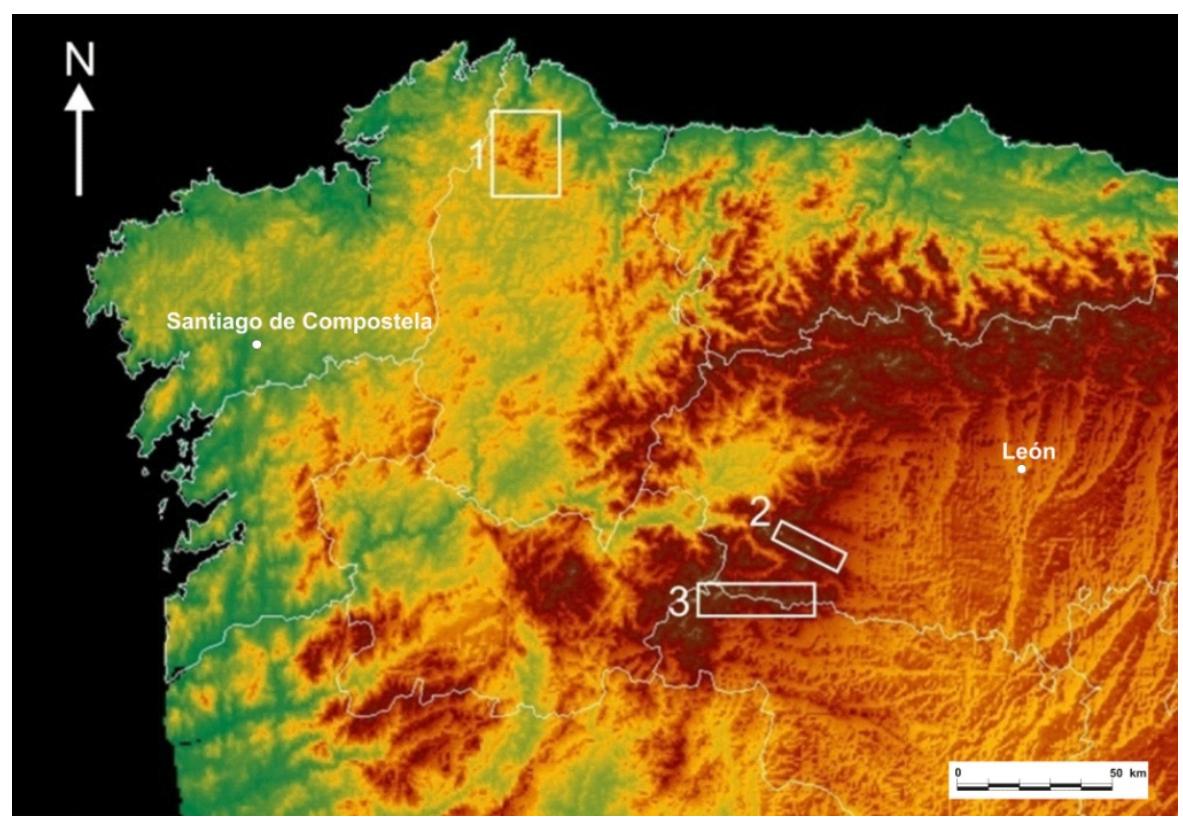

FUENTE.: Los autores.

Figura 6. Mapa litológico de la Serra de Xistral.

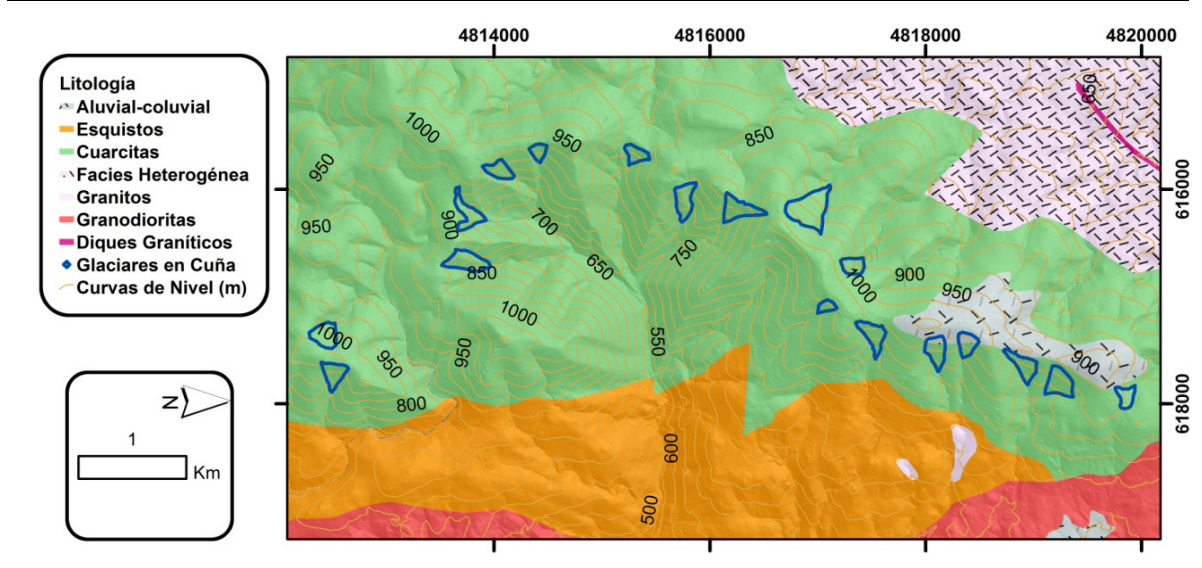

FUENTE.: Los autores. 
Figura 7. Mapa litológico de Teleno.

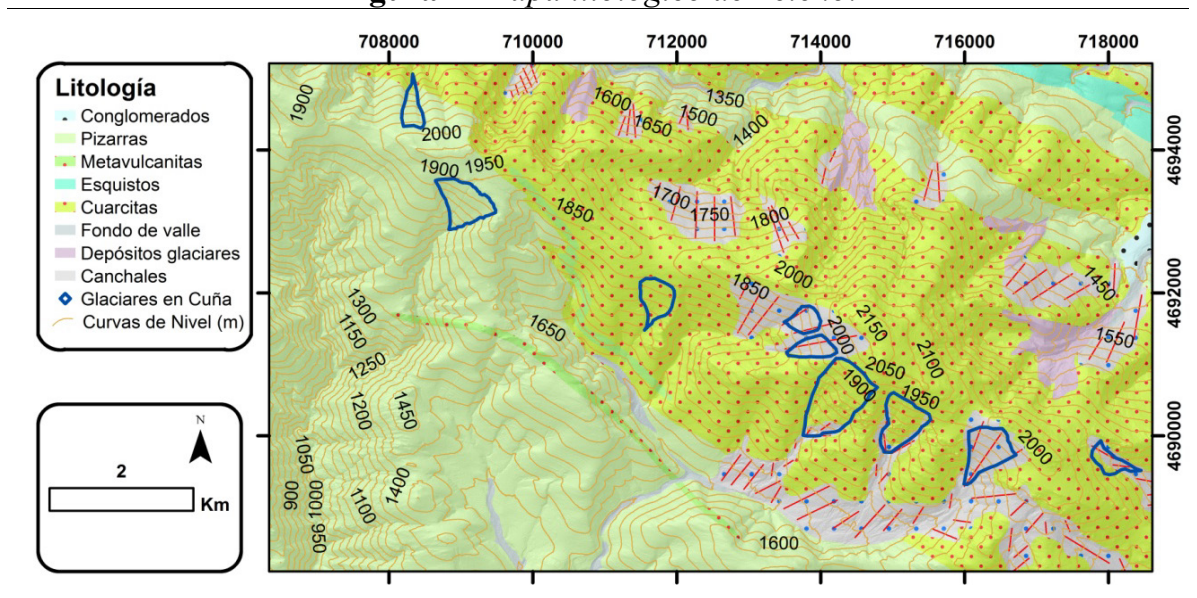

FUENTE.: Los autores.

Figura 8. Mapa litológico de Cabrera.

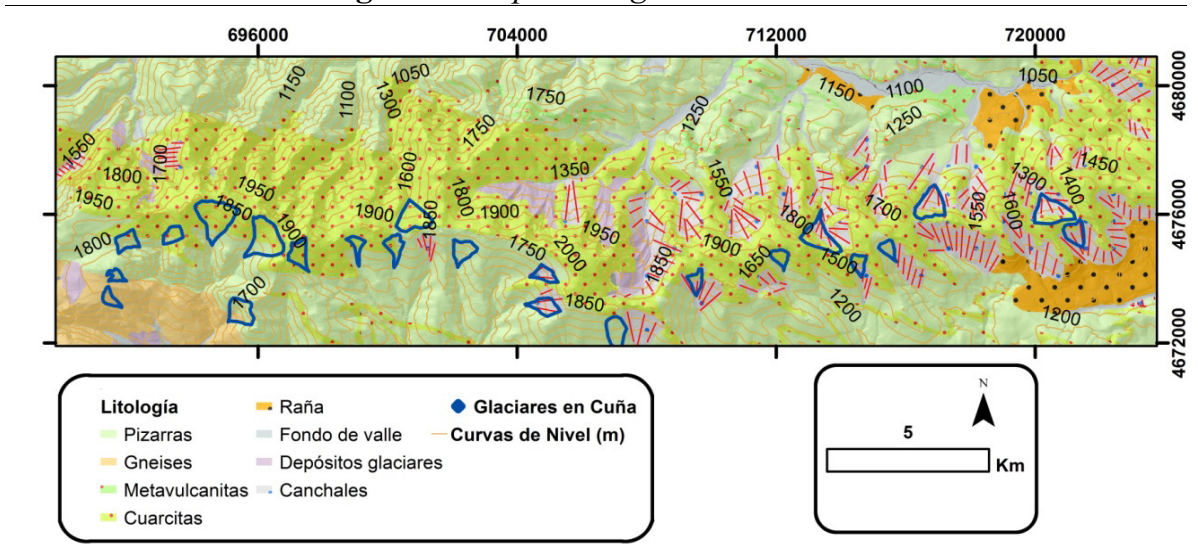

FUENTE.: Los autores.

\section{MODELADO GLACIAR}

Los tres sectores montañosos estudiados presentan una fuerte personalidad desde el punto de vista geomorfológico. Más parecidos entre sí, Teleno y Cabrera son dos alineaciones montañosas de dirección dominante este-oeste, con presencia de superficies somitales bien desarrolladas que rondan los $2.000 \mathrm{~m}$ de altitud, con flancos norte extensos, mientras sus laderas sur son más empinadas. Por su parte, la Serra de Xistral es un conjunto montañoso cercano a la actual línea de costa cuyas cotas máximas, como ya comentamos, superan escasamente los $1.000 \mathrm{~m}$ de altitud. La combinación entre superficies llanas bien 
desarrolladas en las áreas de cumbres y valles de pendiente muy suave en las áreas bajas propiciaron el desarrollo del fenómeno glaciar. En estudios anteriores ya hemos dado noticia de la existencia de los restos evidentes de modelado glaciar en este ámbito (PÉREZ ALBERTI et al., 1993,1995; VALCÁRCEL, 1998; PÉREZ ALBERTI y VALCÁRCEL, 1998). Más recientemente ha quedado de manifiesto que la dimensión de la glaciación, en cuanto a extensión de las masas de hielo, fue incluso mayor de lo descrito en estos trabajos previos (VALCÁRCEL et al., 2015). Se trata por lo tanto de un sector intensamente glaciado. Los glaciares en cuña, descritos ya con detalle en VALCÁRCEL (1998), son formas que parecen corresponder a una fase posterior a este máximo avance reconocido tan extenso, caracterizada por unas condiciones muy frías, pero más secas que la precedente, ya que los glaciares alcanzaron un desarrollo menor (VALCÁRCEL, 1998; PÉREZ ALBERTI y VALCÁRCEL, 1998).

Los glaciares desarrollados en el momento de máximo avance se corresponden a casquetes con prolongación en lenguas (PÉREZ ALBERTI et al., 1993). Se originaron a partir de la acumulación sobre las superficies de cumbres y el descenso de glaciares de «escape» (oulet glacier) que se canalizaron a través de los valles preglaciares de escasa pendiente (VALCÁRCEL, 1998). Por esta razón, las formas de erosión glaciar están poco desarrolladas. No se puede hablar de auténticas artesas glaciares, ya que los hielos se limitaron a arrastrar los materiales preglaciares, acumulándolos en morrenas frontales, laterales y de fondo. Los ejemplos mejores los encontramos en el sector de Rego do Esterco, en el límite sur de la zona glaciada, donde una morrena frontolateral alcanza un desarrollo superior a los cuatrocientos metros de longitud (VALCÁRCEL et al., 2015). Por otra parte, la presencia de una ice cap somital no propició la génesis de auténticos circos y es presumible que sobre las laderas de las cimas más elevadas, como Cuadramón o Xistral, no se produjese la acumulación de hielo suficiente para originar estos. Más bien los hielos se asentaron sobre las superficies aplanadas situadas un escalón más abajo, dejando en resalte a aquellas como nunataks y realizando una labor de conservación del relieve, más que de erosión de este.

Por su parte, el glaciarismo de las Sierras de Teleno son destacables los trabajos de AlONSO OTERO (1982) y más recientemente de LUENGO (2002). En ambos se insiste en la descripción de una glaciación disimétrica, con aparatos bien desarrollados fluyendo hacia el norte, mientras que las laderas orientadas al sur se consideran libres de manifestaciones glaciares. En el caso de la Sierra de La Cabrera, su estudio hay que ligarlo al conjunto del macizo de TrevincaSanabria, del cual es una prolongación. Así, los primeros trabajos son muy tempranos (HALBFASS, 1913; STICKEL, 1929, VOSSELER, 1931, CARLE, 1949, entre otros), pero su posición marginal con respecto al macizo de Trevinca- 
Sanabria posiblemente explique el escaso interés que ha despertado su estudio detallado. Así, solo el trabajo de SCHMITZ (1969) detalla una cartografía de la superficie cubierta de hielo, con dos masas separadas: una la que drena el río Cabrera, y que realmente forma parte del gran icefield de Trevinca-Sanabria y otra independiente que corresponde a la cuenta del Truchillas, siempre mirando a la ladera norte, mientras que la ladera sur quedaría libre de hielos. Más recientemente, en el trabajo de revisión de RODRÍGUEZ RODRÍGUEZ et al. (2015) esta segunda masa más oriental queda reducida a la presencia de siete pequeños glaciares de circo, en su caso prolongados en cortas lenguas. Nuevamente la vertiente sur queda libre de hilos glaciares, según esta interpretación. En estos dos casos los autores describen la glaciación que afectó a estas montañas como de carácter marginal, basándose exclusivamente en el escaso desarrollo longitudinal de los aparatos glaciares, donde se reconocen circos bien definidos, así como aparatos morrénicos laterales o frontolaterales bien desarrollados. En los tres casos, pues, el efecto del modelado glaciar es manifiesto y no se discute. Sin embargo, la amplitud de este, así como su interpretación como «glaciarismo de carácter marginal» puede ser reevaluado a partir de las nuevas observaciones ligadas a la presencia de formas glaciares en cuña y sus subsecuentes formas de erosión.

\section{MATERIAL Y MÉTODOS}

A partir de la experiencia previa sobre otras formas de erosión glaciar (VALCÁRCEL et al., 2013) se digitalizaron las formas breitböden utilizando criterios topográficos, de fotointerpretación y datos de campo. Posteriormente se extrajeron los parámetros morfométricos mediante el análisis 3D de Modelos Digitales de Elevaciones (MDE) con distintas resoluciones. Los parámetros utilizados incluyen factores de altitud, pendiente, orientación y geometría a partir de los cuales se llevó a cabo un análisis estadístico con el objetivo de caracterizar los rangos definitorios de estas formas.

Para el desarrollo de esta metodología partimos de una base cartográfica consistente en los modelos digitales del terreno (con resolución de 5 y $25 \mathrm{~m}$ ) y fotografías aéreas del Instituto Geográfico Nacional, que fue procesada en el sistema de información geográfica ArcGis 10.2 Del MDE se generaron capas raster de pendientes, orientación o acumulación de flujo. Apoyándonos en las fotografías aéreas, en las pendientes y en las acumulaciones se procedió a cartografiar las formas glaciares en cuña. Por un lado, las fotografías aéreas nos permitieron localizar visualmente dónde se encontraban. Por otro lado, las pendientes y las acumulaciones de flujo nos permitieron definir sus límites de manera más objetiva: la pendiente define el límite superior de la forma glaciar en cuña debido a que en ese punto se produce una ruptura de pendiente, mientras que la acumulación de flujo define los límites laterales de la forma glaciar 
en cuña, ya que marca claramente sus acanaladuras laterales. Una vez cartografiados se extrajeron tres tipos de parámetros: ráster, geométricos y cualitativos. Dentro de los datos ráster se diferencian pendientes, elevación y orientación. Dentro de los geométricos fueron generados el alto, el ancho o el área, entre otros, por medio de la herramienta «envelop» para evitar posibles errores humanos en las mediciones. Dentro de los datos cualitativos fueron delimitados disimetría, erosión (posterior a la retirada del hielo) y la presencia o ausencia de depósitos mediante las fotografías aéreas y el trabajo de campo (CUADRO 1). Una vez conseguidos estos datos, se procedió a su análisis y tratamiento en hoja de cálculo (Microsoft Excel, 2007), de los que se obtuvieron valores como el de pendiente media, o como la relación de largo/ancho, entre otros. Siguiendo metodologías propias del cálculo de las direcciones de viento se generaron las gráficas de orientaciones de los glaciares en cuña. Se sigue intentando desarrollar una metodología para cuantificar la erosión que pudieron generar estos glaciares sobre las laderas.

\section{RESULTADOS}

Aplicando la metodología descrita fueron cartografiados 50 glaciares en cuña en los dos sectores (FIGURAS 9, 10, 11): 18 en la $\mathrm{S}^{\mathrm{a}}$. de Xistral y 32 en el complejo Cabrera-Teleno. Podemos diferenciar tres tipos de resultados: los trabajados en la hoja de cálculo, los obtenidos del trabajo de campo y de la fotointerpretación, y los obtenidos del análisis de componentes principales.

Cuadro 1. Parámetros y resultados obtenidos en la Serra de Xistral.

\begin{tabular}{|c|c|c|c|c|c|c|c|c|c|c|c|}
\hline$A$ & $B$ & $C$ & $D$ & $E$ & $F$ & $G$ & $H$ & $I$ & $J$ & $K$ & $L$ \\
\hline BX17 & 452,97 & 205,32 & 2,21 & 752 & 919 & 167 & 32,46 & 13632,52 & 0 & 1 & 1 \\
\hline BX5 & 364,07 & 201,08 & 1,81 & 682 & 866 & 184 & 37,06 & 27156,54 & 1 & 1 & 1 \\
\hline BX7 & 330,73 & 191,82 & 1,72 & 812 & 969 & 157 & 32,78 & 41695,87 & 0 & 1 & 1 \\
\hline BX15 & 296,33 & 182,03 & 1,63 & 809 & 953 & 144 & 34,16 & 40491,55 & 1 & 1 & 1 \\
\hline BX9 & 178,59 & 123,03 & 1,45 & 942 & 1017 & 75 & 28,52 & 43143,8 & 1 & 1 & 3 \\
\hline BX14 & 218,28 & 157,90 & 1,38 & 848 & 970 & 122 & 39,66 & 41756,32 & 1 & 1 & 3 \\
\hline BX6 & 354,81 & 280,99 & 1,26 & 827 & 999 & 172 & 32,64 & 29502,75 & 1 & 1 & 1 \\
\hline BX8 & 239,45 & 191,82 & 1,25 & 845 & 972 & 127 & 31,59 & 53127,35 & 0 & 0 & 0 \\
\hline BX1 & 280,46 & 254,66 & 1,10 & 856 & 1009 & 153 & 34,57 & 36568,66 & 0 & 1 & 0 \\
\hline BX13 & 459,32 & 419,10 & 1,10 & 782 & 979 & 197 & 29,13 & 43360,3 & 0 & 0 & 0 \\
\hline BX11 & 331,79 & 307,98 & 1,08 & 768 & 908 & 140 & 31,67 & 25417,45 & 1 & 1 & 1 \\
\hline BX2 & 173,83 & 167,48 & 1,04 & 852 & 953 & 101 & 34,56 & 39177,25 & 0 & 0 & 1 \\
\hline BX18 & 246,06 & 245,58 & 1,00 & 901 & 1003 & 102 & 27,26 & 50514,21 & 0 & 1 & 3 \\
\hline BX10 & 261,94 & 290,51 & 0,90 & 761 & 881 & 120 & 29,40 & 17482,08 & 0 & 1 & 1 \\
\hline BX3 & 195,26 & 230,98 & 0,85 & 760 & 840 & 80 & 28,45 & 24290,73 & 0 & 1 & 1 \\
\hline BX12 & 183,89 & 218,28 & 0,84 & 818 & 873 & 55 & 19,20 & 49293,19 & 1 & 0 & 0 \\
\hline BX16 & 292,10 & 408,20 & 0,72 & 774 & 931 & 157 & 34,31 & 29826,34 & 1 & 1 & 1 \\
\hline BX4 & 279,40 & 398,46 & 0,70 & 765 & 967 & 202 & 34,88 & 98915,97 & 1 & 1 & 1 \\
\hline
\end{tabular}

A, Código; B, Largo (m); C, Ancho (m); D, Relación L/A; E, Altitud Mínima (m); F, Altitud Máxima (m); G, Rango (m); H, Pendiente Media (grados); I, Área (m²); J, Disimetria; K, Erosión; L, Depositos 
Figura 9. Mapa de pendientes de la Serra de Xistral.

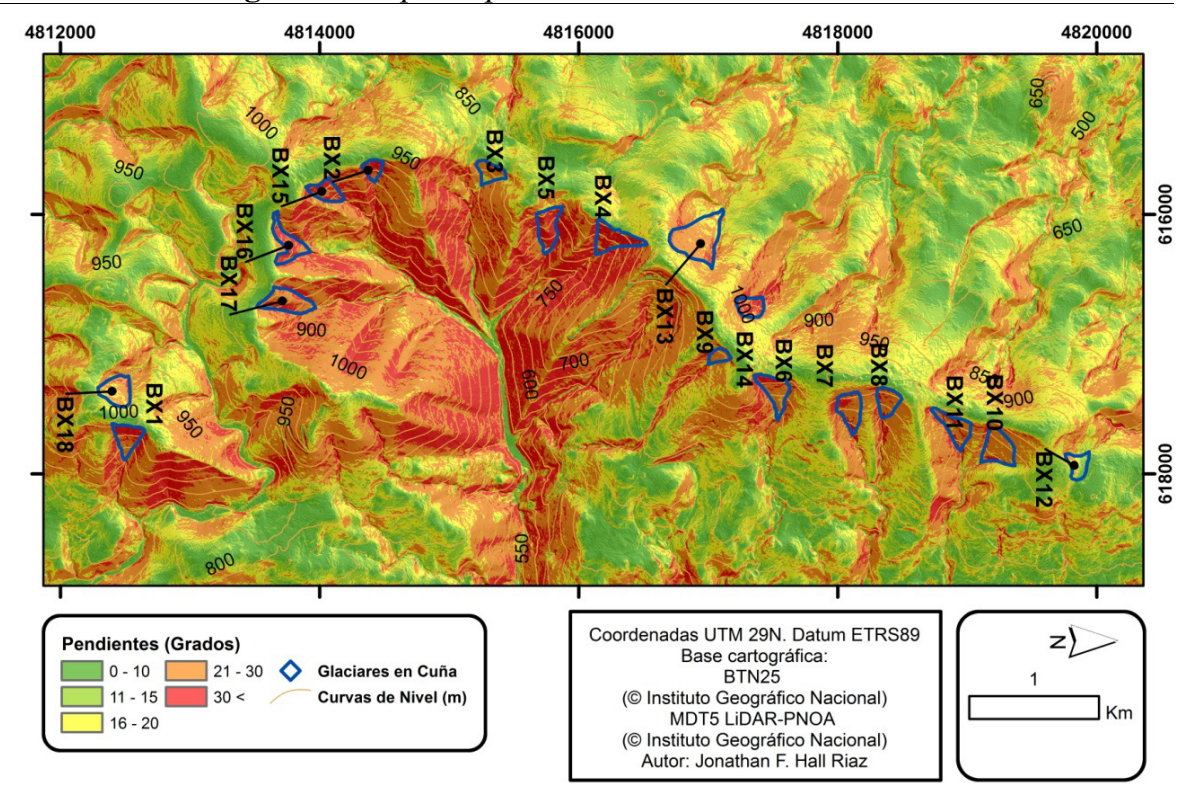

FUENTE.: Los autores.

Figura 10. Mapa de pendientes de Teleno.

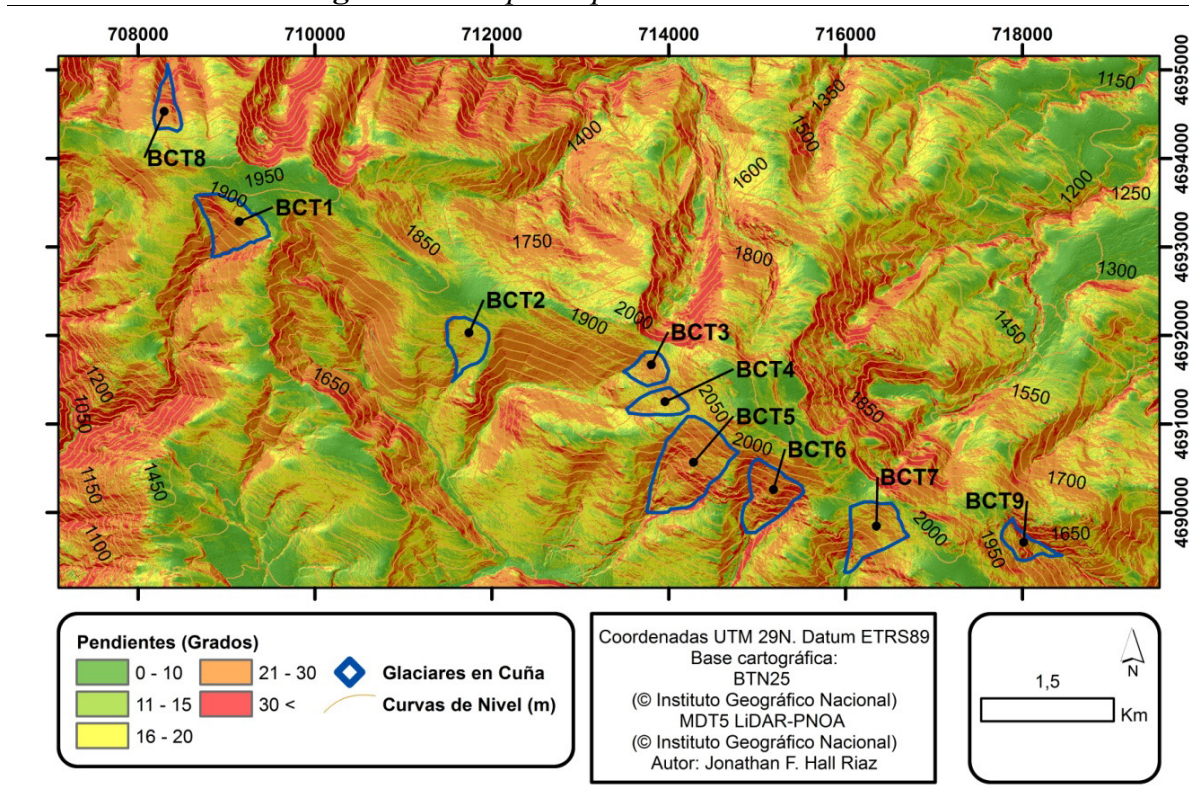

FUENTE.: Los autores. 
Cuadro 2. Parámetros y resultados obtenidos en el complejo Cabrera-Teleno.

\begin{tabular}{|c|c|c|c|c|c|c|c|c|c|c|c|}
\hline$A$ & $B$ & $C$ & $D$ & $E$ & $F$ & $G$ & $H$ & $I$ & $J$ & $K$ & $L$ \\
\hline BCT8 & 756,71 & 317,50 & 2,38 & 1575 & 1928 & 353 & 32,35 & 211277,3043 & 0 & 1 & 1 \\
\hline BCT4 & 704,14 & 313,97 & 2,24 & 1784 & 2013 & 229 & 22,02 & 330691,3256 & 0 & 0 & 2 \\
\hline ВСТ22 & 1088,10 & 565,55 & 1,92 & 1482 & 1827 & 345 & 23,39 & 166277,3805 & 0 & 0 & 2 \\
\hline ВСТ24 & 1237,92 & 654,92 & 1,89 & 1387 & 1828 & 441 & 23,24 & 481107,628 & 0 & 0 & 3 \\
\hline ВСТ23 & 825,50 & 478,90 & 1,72 & 1501 & 1844 & 343 & 30,91 & 349104,2908 & 0 & 1 & 3 \\
\hline ВСТ19 & 1083,89 & 635,00 & 1,71 & 1466 & 1854 & 388 & 26,77 & 329710,8234 & 1 & 1 & 3 \\
\hline ВCT17 & 1051,72 & 641,62 & 1,64 & 1568 & 1973 & 405 & 27,51 & 465076,7888 & 1 & 1 & 3 \\
\hline ВСТ21 & 876,43 & 558,93 & 1,57 & 1631 & 1911 & 280 & 25,82 & 286023,565 & 0 & 0 & 3 \\
\hline BCT11 & 605,90 & 388,94 & 1,56 & 1654 & 1865 & 211 & 26,89 & 700007,7004 & 0 & 0 & 3 \\
\hline ВCT18 & 926,04 & 598,62 & 1,55 & 1490 & 1813 & 323 & 23,92 & 198515,602 & 0 & 1 & 2 \\
\hline BCT2 & 726,02 & 478,37 & 1,52 & 1576 & 1797 & 221 & 20,32 & 232491,9069 & 0 & 1 & 3 \\
\hline ВCT27 & 704,85 & 472,02 & 1,49 & 1398 & 1691 & 293 & 28,27 & 233233,5184 & $\mathbf{0}$ & $\mathbf{0}$ & 2 \\
\hline ВСТ9 & 683,91 & 476,25 & 1,44 & 1607 & 1840 & 233 & 30,92 & 335314,4307 & 1 & 1 & 3 \\
\hline ВСТ28 & 859,90 & 648,23 & 1,33 & 1219 & 1496 & 277 & 26,23 & 166540,9917 & 0 & 1 & 3 \\
\hline ВСТ3 & 510,12 & 387,35 & 1,32 & 1819 & 2003 & 184 & 23,78 & 293896,8972 & 0 & 0 & 2 \\
\hline ВСТ26 & 679,45 & 560,92 & 1,21 & 1370 & 1658 & 288 & 29,65 & 975312,2455 & 0 & 1 & 3 \\
\hline ВCT6 & 833,44 & 701,15 & 1,19 & 1642 & 2006 & 364 & 33,62 & 143725,6459 & 1 & 1 & 2 \\
\hline ВСТ25 & 654,85 & 555,63 & 1,18 & 1458 & 1758 & 300 & 29,59 & 919624,4451 & 1 & 1 & 3 \\
\hline ВСТ20 & 863,20 & 740,83 & 1,17 & 1446 & 1760 & 314 & 24,86 & 356503,998 & 0 & 1 & 3 \\
\hline ВСТ29 & 1059,66 & 948,53 & 1,12 & 1259 & 1611 & 352 & 24,78 & 122651,595 & 0 & 1 & 3 \\
\hline BCT13 & 611,19 & 551,66 & 1,11 & 1531 & 1860 & 329 & 28,47 & 348338,6153 & 0 & 1 & 3 \\
\hline BCT7 & 799,04 & 722,31 & 1,11 & 1683 & 2028 & 345 & 25,51 & 123772,649 & 0 & 1 & 2 \\
\hline ВСТ31 & 1365,25 & 1277,94 & 1,07 & 1390 & 1765 & 375 & 24,03 & 127548,4182 & 1 & 1 & 3 \\
\hline ВCT5 & 1087,44 & 1034,52 & 1,05 & 1567 & 2058 & 491 & 30,91 & 656097,3023 & 0 & 1 & 2 \\
\hline BCT14 & 1377,16 & 1317,63 & 1,05 & 1512 & 2013 & 501 & 24,66 & 201646,9087 & 0 & 1 & 3 \\
\hline BCT16 & 829,47 & 801,69 & 1,03 & 1297 & 1723 & 426 & 36,43 & 194421,4255 & 1 & 1 & 2 \\
\hline ВCT10 & 748,77 & 743,48 & 1,01 & 1554 & 1839 & 285 & 37,38 & 634987,4091 & 0 & 1 & 3 \\
\hline ВCT15 & 1214,44 & 1266,03 & 0,96 & 1520 & 1979 & 459 & 23,15 & 179413,4413 & 0 & 1 & 3 \\
\hline ВCT12 & 597,96 & 650,88 & 0,92 & 1486 & 1801 & 315 & 29,04 & 605981,8697 & 1 & 0 & 0 \\
\hline ВСТ32 & 992,19 & 1135,06 & 0,87 & 1518 & 1828 & 310 & 21,83 & 330071,7847 & 0 & 1 & 3 \\
\hline BCT1 & 711,20 & 833,44 & 0,85 & 1649 & 1943 & 294 & 29,60 & 620338,9423 & 0 & 1 & 2 \\
\hline BCT30 & 928,69 & 1254,13 & 0,74 & 1249 & 1555 & 306 & 22,50 & 124763,9844 & 1 & 1 & 3 \\
\hline
\end{tabular}

A, Código; B, Largo (m); C, Ancho (m); D, Relación L/A; E, Altitud Mínima (m); F, Altitud Máxima (m); G, Rango (m); H, Pendiente Media (grados); I, Área $\left(\mathrm{m}^{2}\right)$; J, Disimetria; K, Erosión; L, Depositos

Dentro del primer grupo destacamos las orientaciones dominantes. En la Serra de Xistral dominan las orientaciones este, mientras que en el complejo Cabrera-Teleno dominan las orientaciones sur (FIGURA 12). Esto se debe a las respectivas orientaciones de las sierras y a la configuración topográfica de las mismas, ya que en las vertientes en las que las laderas no permitieron el desarrollo de glaciares en lengua facilitaron la formación de glaciares en cuña. Estas laderas se corresponden con las más abruptas. También se engloban dentro de este grupo los resultados de dimensiones, donde observamos que los glaciares en cuña de Cabrera y Teleno presentan unas mayores dimensiones, que se reflejan en un mayor rango de altitudes y en valores de largo (450-170 en Xistral, 1400-500 en Cabrera-Teleno) y ancho (420-120 en Xistral, 1.300300 en Cabrera-Teleno) mayores. Los resultados de pendientes varían entre los 20-30 grados y son similares en ambas sierras (CUADROS 1 y 2). Estos valores se ajustan a los descritos por LEHMKUHL en las montañas asiáticas. 
Figura 11. Mapa de pendientes de Cabrera.

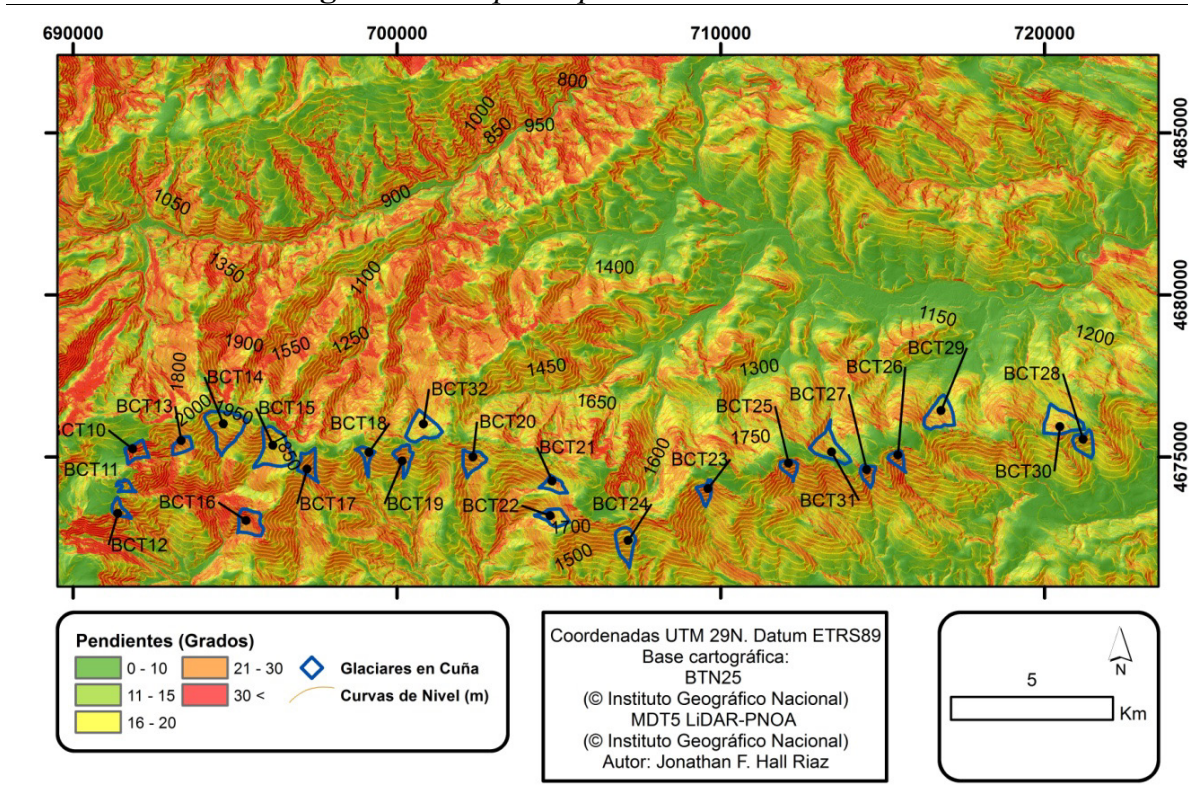

FUENTE.: Los autores.

Figura 12. Orientaciones dominantes de los glaciares en cuña para la Serra de Xistral y el complejo Cabrera-Teleno.

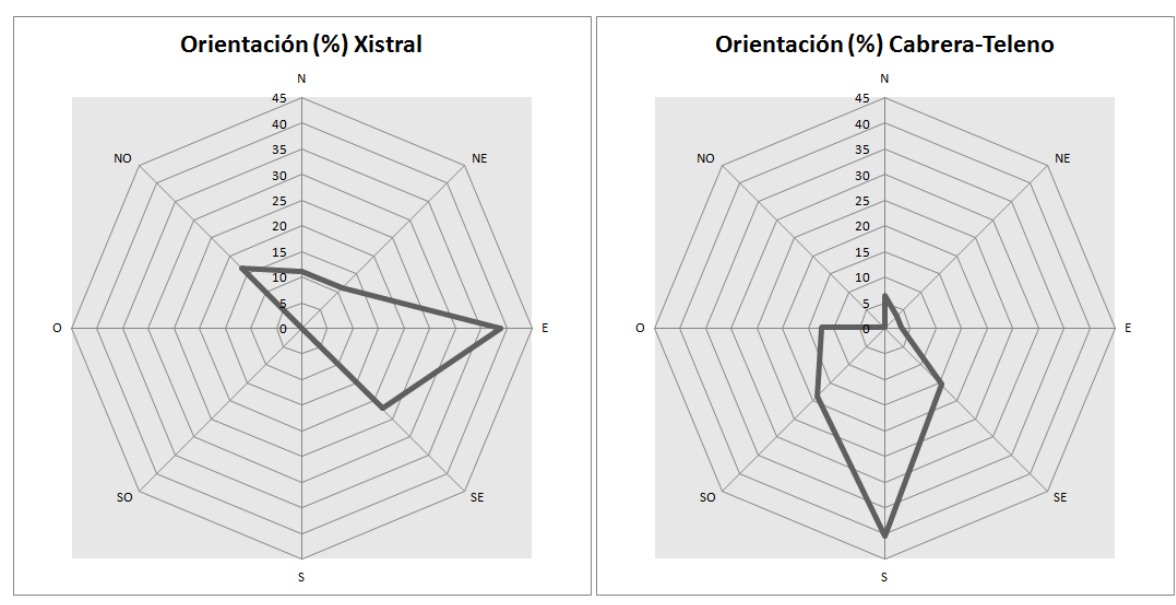

FUENTE.: Los autores.

Dentro del segundo grupo observamos que en la Serra de Xistral presenta una mayor cobertura de sedimentos posterior a la desaparición del hielo, lo que provoca que también presente un mayor número de formas glaciares en 
cuña disimétricas y muestras de erosión fluvial posterior a la desaparición del hielo. Algunas de las muestras de erosión posterior a la desaparición del hielo en Teleno fueron generadas en época romana debido a la minería del oro (MATÍAS, 2006). El complejo Cabrera-Teleno presenta una mayor cobertura de sedimentos en la parte superior de las formas glaciares en cuña.

\section{DISCUSIÓN Y ENCUADRE PALEOCLIMÁTICO}

Desde el punto de vista paleoclimático, la presencia de formas breitböden plantea un dilema. Todos los autores consultados (cf. HÖVERMANN, 1974; HOFMANN, 1992 y LEHMKUHL, 1991) señalan que su existencia, tanto en formas relictas como activas, es indicador de un glaciarismo desarrollado bajo condiciones frío-áridas. Esto contrasta fuertemente con las condiciones actuales de los macizos estudiados, especialmente en el caso de la Serra de Xistral. En ella, además, el glaciarismo correspondiente al máximo avance alcanzó unas dimensiones considerables (VALCÁRCEL et al, 2015), lo que pone en duda que las formas glaciares en cuña se formasen coetáneamente. El máximo avance puede correlacionarse con las fases descritas en otros macizos, especialmente Ancares, donde el máximo avance es temprano (VALCÁRCEL, 1998), anterior al máximo avance global (LGM). Esta interpretación de un máximo avance temprano ha sido corroborada por trabajos recientes (PÉREZALBERTI et. al., 2011, JiMÉNEZ SÁNCHEZ et al., 2013, RODRÍGUEZ RODRÍGUEZ, et al., 2015). En el caso de la Serra de Xistral, únicamente en un momento posterior al máximo avance local de los glaciares es posible plantear la existencia de una segunda fase en la que los glaciares retrocediesen hasta abandonar el fondo de los valles, pero conservando aún pequeñas lenguas de escape o glaciares de pared que modelaron las formas breitböden adosadas a las laderas. Las formas más nítidas se sitúan en el valle del Eume, aunque son reconocibles en otros puntos de la sierra, y su presencia supone dos hechos: que el glaciarismo desarrollado en ese momento requiere unas condiciones de frío intenso y relativa sequedad, y que las masas de hielo activas se restringirían a las áreas más elevadas de la sierra. Se trataría, pues, de un glaciarismo que sólo se podría haber desarrollado bajo las condiciones frías y secas del Pleniglaciar Final (LGM). En estas condiciones todo se encontraría por encima del límite del permafrost continuo, tanto las zonas glaciadas como las no glaciadas. El nivel de nieves permanentes se situaría a cotas muy similares a las descritas para la fase anterior, cercano a los $900 \mathrm{~m}$, pero en este caso el menor desarrollo de los glaciares habría que relacionarlo con la fuerte restricción de humedad y posiblemente con una mayor tasa de insolación en verano (VALCÁRCEL, 1998, VALCÁRCEL et al., 1998). Únicamente en las áreas somitales más propicias se desarrollaría un pequeño domo glaciar que emitiría pequeñas lenguas de escape, a modo de glaciares en cuña. La reconstrucción de 
las paleotemperaturas (VALCÁRCEL, 1998) indica que en estos momentos la Serra de Xistral se encontraba incluida dentro del ámbito de extensión del permafrost discontinuo, y es muy posible que las partes más elevadas estuvieran ocupadas por un cinturón de permafrost continuo, en el cual es factible que se desarrollasen glaciares de base fría como los que serían necesarios para la génesis de las formas glaciares en cuña (VALCÁRCEL, 1998; VALCÁRCEL et. al., 1998).

Por el contrario, la evolución glaciar en las sierras de Teleno y Cabrera pudo ser muy diferente. No se han descrito evidencias de una glaciación más extensa como ocurrió en la Serra de Xistral. En el caso de Teleno se cita una glaciación marginal que afecta solo a la cara norte (ALONSO OTERO, 1982; LUENGO, 2002). Por su parte, la glaciación de la Cabrera puede considerarse marginal con respecto al núcleo principal de Trevinca-Sanabria, pero solo desde este aspecto (SCHMITZ, 1969). En este contexto la coexistencia de los glaciares en cuña con los pequeños glaciares en lengua de las vertientes norte parece verosímil.

En este contexto se puede encontrar una lógica en el análisis de los datos morfométricos. La menor dimensión de las formas descritas en la Serra de Xistral puede deberse al factor litológico-estructural o al factor paleoclimático, o, más posiblemente, a la combinación dialéctica de los dos. Por el contrario, las laderas más abruptas de las vertientes sur de Cabrera y Teleno favorecen desarrollos más largos así como un mayor ensanchamiento de las formas. A ello debemos añadir la posibilidad de una mayor persistencia de la actividad glaciar en las dos últimas, debido a su condición de partida más continentalizada en cualquier contexto glaciar. Esto conllevaría implicaciones paleoclimáticas, como pueden ser las posibles diferencias en el funcionamiento de los gradientes térmicos verticales, tal como ocurre incluso actualmente (MARTÍNEZ CORTIZAS y PÉREZ-AlBerti, 1999).

\section{CONCLUSIONES}

El presente artículo ha sido elaborado consultando cuidadosamente las escasas fuentes existentes en esta materia con la intención de desarrollar una metodología morfométrica que respete y complete la descripción estrictamente morfológica establecida hasta el momento. A lo largo de esta investigación hemos podido observar que la relación L/A no es el único rasgo definitorio de las formas glaciares en cuña. Elementos como la pendiente, la superficie o los procesos ocurridos tras la retirada del hielo juegan un papel importante en la morfometría glaciar actual. En un futuro próximo esperamos poder realizar un trabajo de campo más intensivo en las zonas en las que estos 
glaciares fueron descritos. Aunque desde el principio la intención fue el desarrollo de una metodología SIG que permitiese cubrir grandes superficies de terreno (en zonas especialmente inaccesibles) con poco trabajo de campo. La importancia de esta metodología radica en la intención de alcanzar una mayor comprensión de una forma de erosión glaciar que además es un importante indicador paleoambiental.

\section{AGRADECIMIENTOS}

Los autores quieren manifestar su agradecimiento al Dr. D. Pedro Ibarra Torre (IGME), por la amable cesión de la fotografía de la FIGURA 1. También quieren manifestar su agradecimiento a dos revisores anónimos y a los editores Dr. D. José Cortizo Álvarez y Dr. D. Enrique Serrano Cañadas por su contribución a la mejora del presente manuscrito.

\section{BIBLIOGRAFÍA:}

ALONSO OTERO, F. (1982): «Observaciones sobre la morfología glaciar de la Sierra del Teleno (Montes de León)». Anales de Geografia de la Universidad Complutense, 2, 85-98.

CARLE, W. (1949): «Testigos de una glaciación diluvial en la Galicia española». Estudios Geográficos, 37, 701-706 (traducido de W. CARLE (1947): ZengereinerdiluvialenVereisung in Spanisch-Galicien, Frankfurt Naturen Vok, 122-130).

HaLbFass, W. (1913): «Der Castañedesee, der grösste Süsswasserse Spaniens, und Seine Umgebud». Petermanns Geographische Miteilungen 59 (2), 306-312.

HoFMANN, J. (1992): «Investigations of present and former periglacial, nival and glacial features in Central Helan Shan (Inner Mongolia/Peoples Rep. of China)». Zetschrift für Geomorphologie Suppl.-Bd. 86, 139-154.

HöVERMANN, J. (1974): «Neue Befundezurpleisozän en Harz-Vergletscherung». Abh. d. Braunsch weigischen Wiss. Gelellschaft, 24, 31-52.

JimÉnez SÁnchez, M.; Rodríguez RodríGUeZ, L.; GarCía Ruiz, J. M.; Domínguez Cuesta, M. J.; Farias, P., Valero Garcés, B. y Valcárcel, M. (2013): «A review of glacial geomorphology and chronology in northern Spain: timing and regional variability during the last glacial cycle». Geomorphology, 196, $50-64$.

LEHMKUHL, F. (1991): «Breitböden als glaziale erosions formen einberichtüber vergletscherungstypen im Qilian Shan und im Kunlun Shan (China)». Zeit. Für Gletscherkunde und Glazialgeologie, 27/28, 51-62.

LuENGo Ugidos, M.A., (2002): «El glaciarismo de la Sierra del Teleno (León)». En: Redondo Vega, J.M., Gómez Villar, A., GonzÁlez Gutiérrez, R.B., CARRERA GÓMEZ, P. (Eds.), El modelado de origen glaciar en las montañas leonesas. Universidad de León, León, 29-64.

MARTínez CORTIZAS, A, y PÉrEZ ALBERTI, A (Eds.) (1999): Atlas climático de Galicia. Santiago de Compostela. Xunta de Galicia, $207 \mathrm{pp}$.

MATíAS RodRÍGUEZ, R. (2006): «La minería aurífera romana en el Noroeste de la Hispania: Ingeniería, minería y gestión de las explotaciones auríferas romanas en 
la Sierra del Teleno (León-España)». En Nuevos Elementos de ingeniería Romana. III Congreso de Obras Públicas Romanas. Junta de Castilla y León-Colegio de ITOP, Astorga, 213-266.

PÉreZ-AlBerti A., RodríGuez Guitián, M. A. y VAlCÁrCel, M. (1993): «Las formas y depósitos glaciares en las Sierras Orientales y Septentrionales de Galicia (NW Península Ibérica)». En: PÉREZ AlberTI, A., GuITIÁn RiVERA, L. y RAMIL REGO, P. (Eds.): La evolución del paisaje en las montañas del entorno de los Caminos Jacobeos. Xunta de Galicia. Santiago de Compostela, 61-90.

PÉrez-Alberti A., Rodríguez Guitián, M. A. y VAlCÁRCel, M. (1995): «Acción e importancia del frío durante el cuaternario reciente en las Sierras Septentrionales de Galicia (Noroeste Ibérico)». En Actas III Reunião do Quaternario Ibérico. G.T.P.E.Q.-A.E.Q.U.A. Coimbra, 79-84.

PÉREZ-AlBERTI A. y VALCÁRCEL, M. (1998): «Caracterización y distribución espacial del glaciarismo en el noroeste de la Península Ibérica». En A. GOMEZ ORTIZ y A. PÉREZ Alberti (Eds.): Las Huellas Glaciares de las Montañas Españolas. Servicio de Publicacions da Universidade de Santiago de Compostela, Santiago de Compostela, 17-54.

PéreZ-Alberti, A.; VAlcÁrcel, M.; Martini, P. I.; PAscucci, V. y Andreucci, S. (2011): «Upper Pleistocene glacial valley-junction sediments at Pias, Trevinca Mountains, NW Spain». Geological Society, London, Special Publications, 354(1), 93-110.

Rodríguez Guitián, M. A. y VAlCÁrCel, M. (1994): «Contribución al conocimiento del glaciarismo pleistoceno en la vertiente suroccidental del Macizo de Peña Trevinca (Montañas-Sanabrienses, NW Ibérico)». En A. Gómez Villar, J.M. GARCÍA RUIZ y J. ARNÁEZ (eds.). Geomorfología en España: III Reunión de Geomorfología, Logroño, Vol. 1, 241-252.

RodríGUEZ-RodríGUEZ, L., JimÉnEZ-SÁnCHEZ, M., DomíngueZ-CuESTA, M. J., y ARAMBURU, A. (2015): «Research history on glacial geomorphology and geochronology of the Cantabrian Mountains, north Iberia $\left(43-42^{\circ} \mathrm{N} / 7-2^{\circ} \mathrm{W}\right) »$. Quaternary International, 364, 6-21.

Rost, K. T. (1993): «Die jungpleistozäne Vergletscherung des Qinling Shan (Provinz Shaanxi): Ein Beitragzur Vergletscherungs problematik ostchinesischer Gebirge (A Contribution to the Problem of Pleistocene Glaciations in the Mountains of East-China)». Erdkunde, 131-142.

Rost, K.T. (1994): «Paleoclimatic field studies in and along Qinling Shan (Central China)». Geojournal, 34(1), 107-120.

Rost, K. T. (2000): «Pleistocene paleoenvironmental changes in the high mountain ranges of central China and adjacent regions». Quaternary International, 65, 147 160 .

SCHMITZ, H., (1969): «Glazial morphologische Untersuchungenim Bergland Nordwestspaniens (Galicien/León)». Kölner Geographische Arbeiten, 23, 1-157.

SCHNEIDER, H-J. (1963): «Die Gletschertypen Versuchim Sinneeinereinhetlichen Terminologie». Geogr. Taschenbuch. Weisbaden, 1962/63, 276-283.

STICKEL, R. (1929): «Observaciones de morfología glaciar en el Noroeste de España». Bol. de la Real Soc. Esp. de Historia Natural, 29, 297-313. 
VALCÁRCEL, M. (1998): Evolución geomorfológica y dinámica de las vertientes en el $N E$ de Galicia. Importancia de los procesos de origen frío en un sector de las montañas lucenses. Tesis Doctoral. Universidade de Santiago de Compostela. Inédita. $395 \mathrm{pp}$.

VAlCÁRCEL, M. y PÉREZ-AlberTI, A. (1998): «Estimación de las paleotemperaturas en Galicia a partir de los datos geomorfológicos y climáticos». En A. GóMEZ ORTIZ y F. Salvador Franch (edss). Investigaciones recientes de la geomorfología española. Servei de gestió i evolució del paisatge, Vicerectorat de Recerca/Geoforma Ediciones. Granada, 767-770.

Valcárcel, M., Otero Otero M.; Martínez Cortizas A. y Blanco ChaO, R. (2013): «Glacial and paraglacial dynamics inside glacial cirques. A 3D morphometric analysis». En 8th International Conference (IAG) on Geomorphology, Paris, pp. 1005.

Valcárcel, M., Pontevedra Pombal, X., Sampedro, J.R., García Rodeja, E., Blanco ChaO, R. y Costa CASais, M. (2015): «Las Sierras Septentrionais de Galicia: Un patrimonio europeo singular; geomorfología». En A. HILARIO, M. Mendia, M. Monge-Ganuzas, E. Fernández, J. Vegas y A. Belmonte (eds.). Patrimonio geológico y geoparques, avances de un camino para todos. Cuadernos del Museo Geominero, 18. IGME, Madrid, 137-142.

Vosseler, P. (1931): «Eiszeitstudien im nordweslichen Spanien». Zeits. Für Gletscherkunde, 89-104.

WiLHELM, F. (1975): Schnee-und Gletscherkunde. Berlin. Walter de Gruyter. 434 pp. 